\title{
On path dependent loss and switch crosstalk reduction in optical networks
}

\author{
Rza Bashirov*, Tolgay Karanfiller \\ Department of Applied Mathematics and Computer Science, Eastern Mediterranean University, Famagusta, North Cyprus, via Mersin-10, Turkey
}

\section{A R T I C L E I N F O}

\section{Article history:}

Received 9 March 2009

Received in revised form 29 August 2009

Accepted 6 November 2009

\section{Keywords:}

Optical interconnection network

Path dependent loss

Switch crosstalk

Permutation admissibility

P/T-net

P-invariants

\begin{abstract}
A B S T R A C T
Although optical multistage interconnection networks (OMINs) promise to meet the ever growing demands of communication networks and multiprocessor systems in fast communication, they suffer from challenges such as path dependent loss and switch crosstalk. In this paper, we propose an innovative approach for reducing not only the path dependent loss but also the number of switch crosstalks in OMINs. Our approach is centered upon modelling OMINs with Petri nets and using the P-invariants method to determine the minimum number of stages $m_{\min }$ that is sufficient to establish requested communication patterns in variable-stage OMINs. Being composed of the smallest number of stages and consequently directional couplers (or photonic switches), $m_{\min }$-stage OMIN employs minimal structure and, therefore, path dependent loss and also number of switch crosstalks reach the least possible values in the realization of requested communication patterns.

We prove that the size of Petri nets created in this work is in polynomial dependence on the problem size which alleviates memory consumption significantly and ascertains the fact that memory capacity and performance of modern computers are indeed sufficient to run our task. We also show that the complexity results obtained in this research improve similar results reported in our previous paper. We carry out a series of computer experiments to confirm the effectiveness of the proposed approach.
\end{abstract}

(c) 2009 Elsevier Inc. All rights reserved.

\section{Introduction}

The two major problems arising in optical communication are path dependent loss and switch crosstalk. Optical signals usually become weak after passing through a long connection path, which may potentially cause signal distortion. This phenomenon is known as path dependent loss or attenuation [11,16,23]. In a large OMIN, a substantial part of this path dependent loss is directly proportional to the number of directional couplers along an optical path, which is determined by the architecture used and the network size [7,13]. Pass dependent loss in effect leads to such unwanted factors as an increase of power consumption in the system and errors in transmission of optical signals. Being a problem of primary interest in optical communication, pass dependent loss is still very much in the focus of researchers.

Another problem arising in OMINs is switch crosstalk - a situation when two paths sharing a directional coupler experience unwanted coupling from one path to the other $[8,22]$. Switch crosstalk is the most significant factor which reduces clarity of an optical signal, limits the size of an optical network and leads to error rate degradation.

This work deals not only with path dependent loss but also with switch crosstalk in OMINs. The total path dependent loss in any OMIN is the sum of path dependent losses in all directional couplers. This is also true for the total number of switch crosstalks in an OMIN. In order to minimize the total path dependent loss and/or the total number of switch crosstalks in an OMIN with a specified network topology, we determine the minimal number of stages $m_{\min }$, and consequently the minimal

\footnotetext{
* Corresponding author. Tel.: +90 392 6301005; fax: +90 3923651604.

E-mail addresses: rza.bashirov@emu.edu.tr (R. Bashirov), tolgay.karanfiller@emu.edu.tr (T. Karanfiller).
} 
number of directional couplers in the OMIN, that would be sufficient to generate a requested communication pattern. Once $m_{\min }$ is known, a requested communication pattern can be established in the given OMIN with minimal path dependent loss and a minimal number of switch crosstalks. In the present research this is achieved by representing each communication pattern as a permutation of OMIN's inputs into its outputs, and checking 1-through ( $2 n-1)$-stage OMINs employing the same network topology for permutation admissability.

We say that a permutation $\pi: 2^{n} \rightarrow 2^{n}$ is admissible to $2^{n} \times 2^{n}$ OMIN if there exists a setting of the directional couplers in the OMIN such that the input $i$ is connected to the output $\pi(i)$ for all $i$. Admissibility of an arbitrary permutation to $m$-stage $2^{n} \times 2^{n}$ OMIN turns out to be equivalent to $2^{m}$-colorability in graphs [20], which is an NP-hard problem. We decided on permutation admissibility by exploiting Petri nets and consequently reducing permutation admissibility to marking reachability in $\mathrm{P} / \mathrm{T}$-nets (place/transition nets). We use P-invariants to perform reachability analysis in $\mathrm{P} / \mathrm{T}$-nets.

We prove that the size of $\mathrm{P} / \mathrm{T}$-nets created in the present work demonstrate polynomial dependence on the OMIN size, which alleviates memory consumption to a significant degree. Moreover, these P/T-nets are more compact compared to the ones obtained in previous work [1], which is an improvement on the complexity results obtained in [1]. It is known [9] that the use of P-invariants for general P/T-nets may lead to spurious solutions. For acyclic P/T-nets, however, the use of P-invariants leads to an irrefragable answer to the question of whether a marking is reachable from the initial marking [12]. P/T-nets obtained in current research employ an acyclic structure indicating that the method of P-invariants is an exact characterization of the set of reachable markings.

The paper is organized as follows. Section 2 reviews key work, to date, in this field. Section 3 deals with common issues in optical communication. Section 4 provides a succinct background on $\mathrm{P} / \mathrm{T}$-nets and P-invariants. The main results are described in Sections 5-8. In Section 5 we create P/T-net models of $2 \times 2$ directional coupler and $2^{n} \times 2^{n} m$-stage OMIN. Section 6 deals with a feasibility analysis of the proposed approach. Section 7 presents and analyzes the results of computer experiments. Finally, conclusions are outlined in Section 8.

\section{Related work}

Approaches that are currently employed or have been proposed for avoiding switch crosstalk can be classified into three categories: space division multiplexing (SDM), time division multiplexing (TDM) and wavelength division multiplexing (WDM). All three approaches have been extensively investigated by many researchers, though each still pose some problems and questions $[3,16,22,25]$. For instance, SDM is far from a cost effective approach, viz. it requires more than double the original OMIN hardware to achieve the same permutation capability. It is customary for the TDM approach to generate a permutation in $n$ passes instead of a single pass, which affects the time-effectiveness of the TDM approach. Finally, the role of WDM in switching, with or without wavelength conversion, is unclear and is a subject of further research.

Much research has also been done on developing directional couplers with reduced path dependent loss [22]. Most of this work is based on the implementation of novel physical-level achievements in optical communication. Attempting to solve path dependent loss by minimizing the number of stages in an OMIN was suggested in [4]. The main result obtained in [4] is a necessary condition, which is also a sufficient condition for special BPC (bit permute complement) permutations, so that a permutation must be admissible to an $m$-stage $2^{n} \times 2^{n}$ OMIN employing the shuffle-exchange interstage communication pattern for $n<m \leqslant 2 n-1$. It has also been shown that, for $1 \leqslant m \leqslant 2 n-1$, the minimum number of stages required to pass a permutation through shuffle-exchange OMIN can be determined in $O\left(2^{n} n\right)$ time. For a BPC permutation this can be done in $O\left(n^{2}\right)$ time. The minimum number of stages needed to perform a permutation can be determined in $O\left(2^{n} n \log n\right)$ time. An $O\left(2^{n} n\right)$ algorithm that determines whether a permutation is admissible to $2^{n} \times 2^{n}$ multistage cube-type networks (MCTNs) was introduced in [20]. In [18] this result was extended to $k$-extra stage MCTN with $k=1$. In the same paper it was shown that a permutation is admissible to a $k$-extra stage MCTN if, and only if, the conflict graph is $2^{k}$-colorable. NP-completeness of the $2^{k}$-coloring problem in graphs, for $k>1$, does not allow us to develop a general method for analysis of the permutation admissibility with polynomial dependence on the number of extra stages. Although there exist efficient algorithms for checking the permutation admissibility for $k=0$ and $k=1$, no such algorithm is known for $k>1$ [4]. The admissibility of frequently used permutations that belong to BP (bit permute), BPC (bit permute complement), LIN (linear combination) and LC (linear combination complement) classes was investigated in [17,19,21]. A comprehensive review of existing survivability schemes in multi-domain optical networks is presented in [6].

Petri nets are a mathematical modelling tool that has been successfully implemented in scientific, engineering and industrial domains. Research in Petri nets falls in two categories: (i) design and analysis of a Petri net that is extended with new characteristics, e.g., soundness-preserving reduction rules for reset workflow nets [24], (ii) using existing Petri nets to problem solve, such as the implementation of Petri nets in E-commerce [5], application of fuzzy Petri nets in supervised learning environments [14], etc.

In [2] we analyzed permutation capability of MINs through reducing this problem to marking reachability in CP-nets, and performing model checking in related CP-nets. In the previous work [1] we demonstrated that CP-nets can also be successfully implemented in verification of the permutation admissability. To accomplish the task we first created a CP-net model, then unfolded this model into an equivalent $\mathrm{P} / \mathrm{T}$-net, with a further optimization of the complete unfolding, and finally used P-invariants to decide on permutation admissibility in the associated P/T-net. 


\subsection{Contributions}

The approach suggested in this paper differs from existing ones in two respects. Firstly, the traditional approaches explore advances in the photonic industry to cope with path dependent loss and/or switch crosstalk. The main idea behind those approaches is to reduce path dependent loss and the number of switch crosstalks by implementing technologically more mature directional couplers. In the present research, however, this is done through designing an optimal OMIN architecture that is composed of a minimum number of directional couplers. We reduce path dependent loss and the number of switch crosstalks in the system by keeping the number of directional couplers as small as possible.

Secondly, to the best of our knowledge, this is the first attempt to adopt Petri nets as a formalism and as a tool in the context of photonic switching. Such research contributes to both fields. In the present paper, we examine 1- through $m$-stage $(1 \leqslant m<2 n-1)$ OMIN for permutation admissibility and thus find the minimal number of stages needed for establishing a desired communication pattern. Essential phases of this technique include creating a P/T-net model directly from OMIN specifications and performing reachability analysis with P-invariants. Avoidance of CP-net modelling, unfolding and optimization phases in the present research leads to the refinement of memory and run time constraints compared to those obtained in [1].

\section{Common issues in optical communication}

There are two choices for optical communication: all-optical and hybrid optical approaches. All-optical switching, where both the controlling and the controlled signals are optical, is seemingly a long-term research project [13]. The hybrid optical approach is based on the use of optoelectronic technology where the signals are optical but the control over the optical signals is carried out electronically. Despite its advantages the hybrid optical approach suffers from path dependent loss and optical crosstalk.

\subsection{Path dependent loss}

Total loss in any optical link is the sum of the losses in all-optical components: propagation loss through the waveguide in a directional coupler (or pass dependent loss), signal loss at waveguide bends, signal loss at waveguide crossovers, propagation loss in the medium, and fiber-to-substrate and substrate-to-fiber coupling loss. As it was reported [16,22], propagation loss through the waveguide among these losses is of major concern and is proportional to the number of directional couplers that the optical signal passes through.

\subsection{Optical crosstalk}

Optical crosstalk arises in one of two forms: channel crossover (or waveguide crossover) and switch crosstalk. The former occurs when the channels carrying the signals cross each other in order to follow-up a specified interstage communication pattern, while the latter arises when two paths sharing a directional coupler experience unwanted coupling from one path to the other. It has also been reported that the number of channel crossovers can be reduced through carefully selecting a suitable planar layout for network topology [10]. It has been shown through a series of experiments [15] that by changing intersection angles between the crossing channels it is possible to keep the channel crossover negligibly small. The switch crosstalk, however, is more severe and becomes one of the major issues in designing OMINs [22].

The four possibilities of switch crosstalk within a directional coupler are illustrated in Fig. 1. In this figure a dashed line is used to indicate the path a switch crosstalk propagates through. Switch crosstalk occurs either between the upper straight

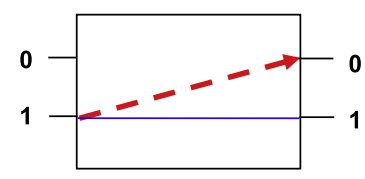

(a) switch crosstalk caused in straight state

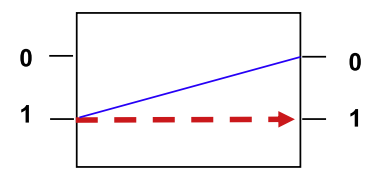

(c) switch crosstalk caused in cross state

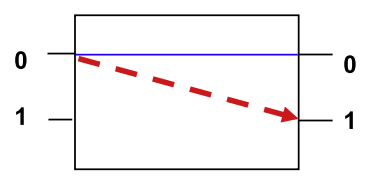

(b) switch crosstalk caused in straight state

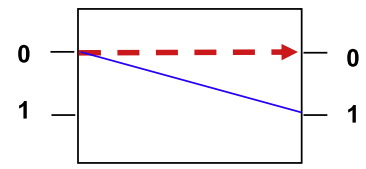

(d) switch crosstalk caused in cross state

Fig. 1. Coupling from (a) lower straight path; (b) upper straight path; (c) lower to upper cross path; and (d) upper to lower cross path. 
path (from input 0 to output 0 ) and upper to lower cross path (from input 0 to output 1 ) or between the lower straight path (from input 1 to output 1 ) and lower to upper cross path (from input 1 to output 0 ).

\section{Theoretical underpinning}

A P/T-net is a 5-tuple, $P N=\left(\mathscr{P}, \mathscr{T}, \mathscr{A}, \mathscr{W}, \mathscr{M}_{0}\right)$ where $\mathscr{P}$ is the finite set of places, $\mathscr{T}$ is the finite set of transitions, such that $\mathscr{P} \cap \mathscr{T}=\emptyset$ and $\mathscr{P} \cup \mathscr{T} \neq \emptyset, \mathscr{A} \subseteq(\mathscr{P} \times \mathscr{T}) \cup(\mathscr{T} \times \mathscr{P})$ is the finite set of $\operatorname{arcs}, \mathscr{W}: \mathscr{A} \rightarrow \mathbb{N}$ is the weight function, and $\mathscr{M}_{0}: \mathscr{P} \rightarrow \mathbb{N}$ is the initial marking.

For a P/T-net with $n$ transitions and $m$ places, the incidence matrix $A=\left[a_{i j}\right]$ is an $n \times m$ matrix with integer entries defined as $a_{i j}=a_{i j}^{+}-a_{i j}^{-}$where $a_{i j}^{+}=w(i, j)$ is the weight of the arc from transition $i$ to its output place $j$ and $a_{i j}^{-}=w(j, i)$ is the weight of the arc to transition $i$ from its input place $j$. Each invariant in a P/T-net can be expressed as the system of linear algebraic equations called state equation

$$
A^{T} \cdot x=M_{d}-M_{0}
$$

where $x$ is an $n \times 1$ column vector of nonnegative integers and is called the firing count vector, $M_{0}$ is the initial marking, and $M_{d}$ is the destination marking. The $i$ th entry of $x$ denotes the number of times that transition $i$ must fire to transform $M_{0}$ to $M_{d}$. It has been reported in [12] that for acyclic P/T-net, $M_{d}$ is reachable from $M_{0}$, if, and only if, there exists a nonnegative firing count vector $x$ satisfying the state Eq. (1).

\section{Model development}

In this section we develop a P/T-net model of a $2 \times 2$ directional coupler and a $2^{n} \times 2^{n} m$-stage OMIN, explain the meaning of P/T-net components and describe the relationship between P/T-net components and OMIN objects.

Definition 1. A P/T-net of $2 \times 2$ directional coupler is a 5 -tuple $\mathrm{PTNCW}_{2 \times 2}=\left(\mathscr{P}, \mathscr{T}, \mathscr{A}, \mathscr{W}, \mathscr{M}_{0}\right)$, such that

- $\mathscr{P}=\mathscr{P}_{\text {in }} \cup \mathscr{P}_{\text {out }}$, where $\mathscr{P}_{\text {in }}=\{$ in0, in 1$\}$ is the set of input places, $\mathscr{P}_{\text {out }}=\{$ iniout $j\}, 0 \leqslant i, j \leqslant 1$ is the set of output places;

- $\mathscr{T}=\{$ straight, cross $\}$ is the set of transitions;

- $\mathscr{A}= \begin{cases}\text { (ini, straight) or (ini, cross), } & \text { for } i=0,1 \\ \text { (straight, iniout } i), & \text { for } i=0,1 \\ \text { (cross, iniout } j), & \text { if } j=1-i \text { and } i=0,1 \\ \text { empty, } & \text { otherwise }\end{cases}$

- $\mathscr{W}=\{1$ : for all $a \in A\}$

- $\mathscr{M}_{0}= \begin{cases}1, & \text { if } p=\text { in } i \text { for } 0 \leqslant i \leqslant 1 \\ 0, & \text { otherwise. }\end{cases}$

The P/T-net diagram in Fig. $2 \mathrm{a}$ is $\mathrm{PTNSW}_{2 \times 2}$ set to the initial state. Transitions straight and cross are used to set the $2 \times 2$ directional coupler to straight state (see Fig. 2b) and cross state (see Fig. 2c), respectively. Places in0 and in1 represent inputs of the $2 \times 2$ directional coupler. Places in 0out 0 and in1out0, that are covered by the left dashbox, represent the upper output of

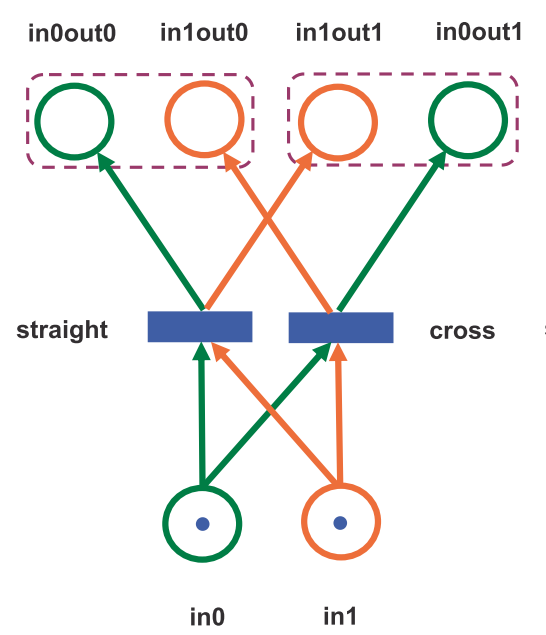

(a)

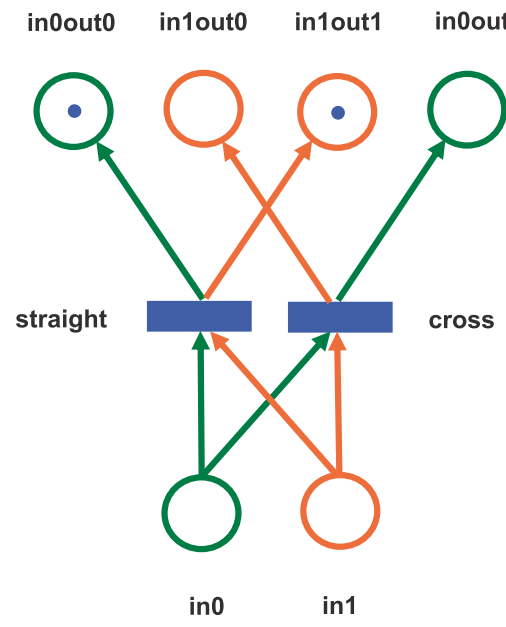

(b)

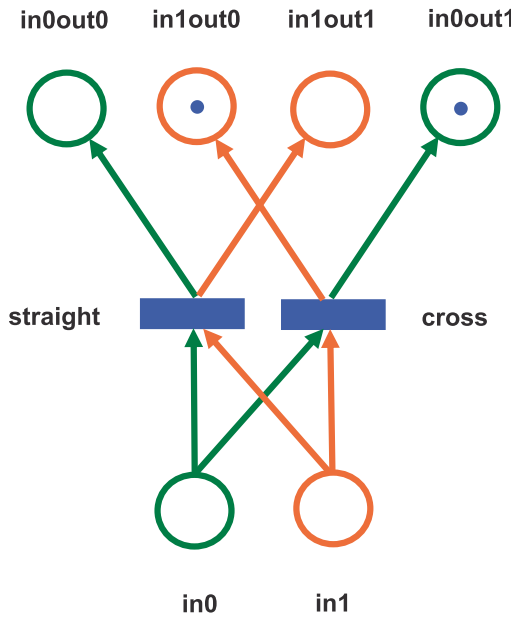

(c)

Fig. 2. P/T-net of $2 \times 2$ directional coupler in: (a) the initial state; (b) straight state; and (c) cross state. 
the $2 \times 2$ directional coupler. Only one of these places can contain a token at a time, indicating which input is linked to the upper output. Likewise places in0out1 and in1out1 (inside the right dashbox) stand for the lower output. A token in any of these places specifies a particular input that is linked to the lower output. PTN $\mathrm{SW}_{2 \times 2}$ is a P/T-net of minimal structure, which fully exhibits the characteristics of the $2 \times 2$ directional coupler.

Definition 2. A P/T-net of $2^{n} \times 2^{n} m$-stage optical network is a 5-tuple PTNOMIN $2^{n}, m=\left(\mathscr{P}, \mathscr{T}, \mathscr{A}, \mathscr{W}, \mathscr{M}_{0}\right)$, such that

- $\mathscr{P}=\mathscr{P}_{1} \cup \cdots \cup \mathscr{P}_{m+1}$, where $\mathscr{P}_{k}=\left\{p_{1}^{(k)}, \ldots, p_{2^{k-1} N}^{(k)}\right\}$ if $1 \leqslant k<n$ and $\mathscr{P}_{k}=\left\{p_{1}^{(k)}, \ldots, p_{2^{n-1} N}^{(k)}\right\}$ if $n \leqslant k<2 n-1$;

- $\mathscr{T}=\mathscr{T}_{1} \cup \cdots \cup \mathscr{T}_{m}$, where $\mathscr{T}_{k}=\left\{t_{1}^{(k)}, \ldots, t_{4^{k-1} N}^{(k)}\right\}$ if $1 \leqslant k<n$ and $\mathscr{T}_{k}=\left\{t_{1}^{(k)}, \ldots, t_{4^{n-1} N}^{(k)}\right\}$ if $n \leqslant k<2 n-1$;

- $\mathscr{A} \subset \bigcup_{k=1}^{m}\left(\mathscr{P}_{k} \times \mathscr{T}_{k}\right) \cup\left(\mathscr{T}_{k} \times \mathscr{P}_{k+1}\right)$;

- $\mathscr{W}=\{1$ : for all $a \in \mathscr{A}\}$;

- $\mathscr{M}_{0}= \begin{cases}1, & \text { if } p \in \mathscr{P}_{1} \\ 0, & \text { otherwise. }\end{cases}$

In PTNOMIN $2^{n}, m$ places and transitions are inherently arranged on alternating levels with the places at the undermost level representing input terminals of OMIN, and places at the topmost level standing for its output terminals (see Fig. 4). According to Definition 2, the arcs are between net components belonging to neighboring and directed towards the topmost, levels. An occurrence of a maximum number of transitions on the $k$ th level sets all directional couplers in $k$ th stage of OMIN. Then transitions on level $k+1$ may occur. This continues until transitions on the last (topmost) level become enabled. The occurrence of a maximum number of transitions on the topmost level sets the P/T-net to a dead marking. Each dead marking corresponds to a particular permutation being established between OMIN's input terminals and its output terminals.

We say an occurrence sequence in $\mathrm{PTNOMIN}_{2^{n}, m}$ is complete if it is of length $m$. In order to keep the structure of the P/T-net as simple as possible we do not impose any predefined order for the occurrence of transitions. Given the specified setting (either straight or cross) of directional couplers in OMIN, all complete occurrence sequences in PTNOMIN $2^{n}, m$ are equivalent and, in fact, they result in the same dead marking, $\mathscr{M}_{d}$. Without the loss of generality we can represent a complete occurrence sequence as follows:

$$
\sigma_{i_{1}, \ldots, i_{m}}=\mathscr{M}_{0}\left[\mathscr { S } _ { 1 } \succ \mathscr { M } _ { 1 } \left[\mathscr { S } _ { 2 } \succ \cdots \mathscr { M } _ { m - 1 } \left[\mathscr{S}_{m} \succ \mathscr{M}_{d}\right.\right.\right.
$$

where $\mathscr{S}_{k} \subset \mathscr{T}_{k}$ for $1 \leqslant k \leqslant m$ and $\mathscr{M}_{d}$ is dead marking.

An example of PTNOMIN $2^{n}, m$ with $n=2$ and $m=2$ (see Fig. 3 ) is shown in Fig. 4 . In this figure, outputs that can be reached by a particular input terminal are represented by unicolored P/T-net fragments. For instance, green places in Fig. 4 indicate the outputs of OMIN (see Fig. 3) that are reachable from input terminal 0, and green arcs correspond to possible routes between input terminal 0 and designated outputs.

\subsection{Modelling an OMIN topology}

It should be stressed that arc determination is the most difficult stumbling block in Definition 2. In fact, we defined an arc to be an element of relation in $\mathscr{P}_{k} \times \mathscr{T}_{k}$ or $\mathscr{T}_{k} \times \mathscr{P}_{k+1}$, and left unanswered the question of how to state precisely which place is linked to which transition or, vice versa. Since we are aiming to build a P/T-net directly from OMIN specifications we need to develop an explicit way of drawing the arcs.

In PTNOMIN $2^{n}, m$, we distinguish between pattern arcs and switch arcs. A pattern $\operatorname{arc}(p, t) \in \mathscr{P}_{k} \times \mathscr{T}_{k}$ is incident from $p$ to $t$ and depends highly on OMIN topology. More precisely, pattern arcs exhibit an effect of interstage communication patterns on a P/T-net. They are created according to the permutations induced by interstage communication patterns. A switch arc $(t, p) \in \mathscr{T}_{k} \times \mathscr{P}_{k+1}$ is incident from $t$ to $p$ no matter which communication pattern is chosen to link neighboring stages in OMIN.

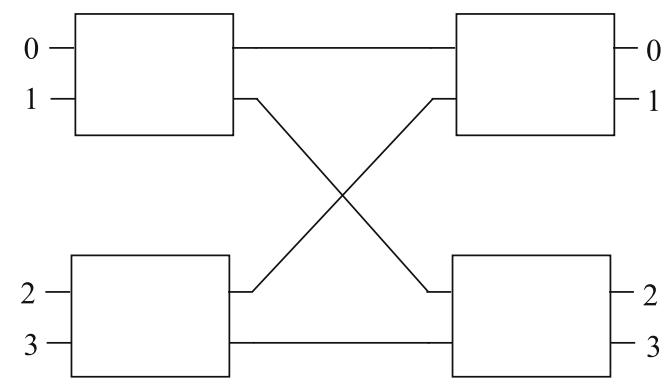

Fig. 3. $4 \times 42$-stage OMIN made of $2 \times 2$ directional couplers. 


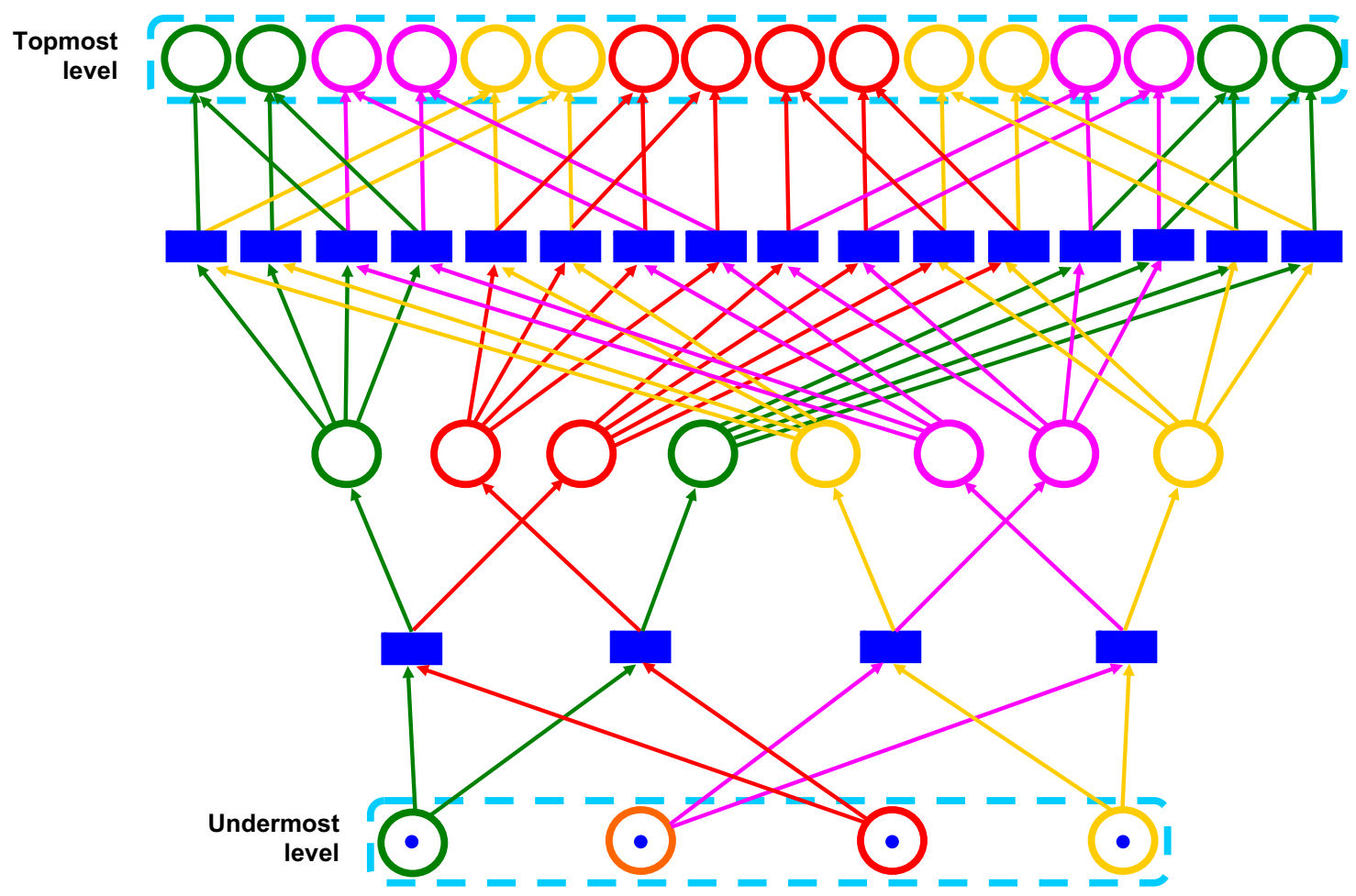

Fig. 4. P/T-net of $4 \times 42$-stage OMIN.

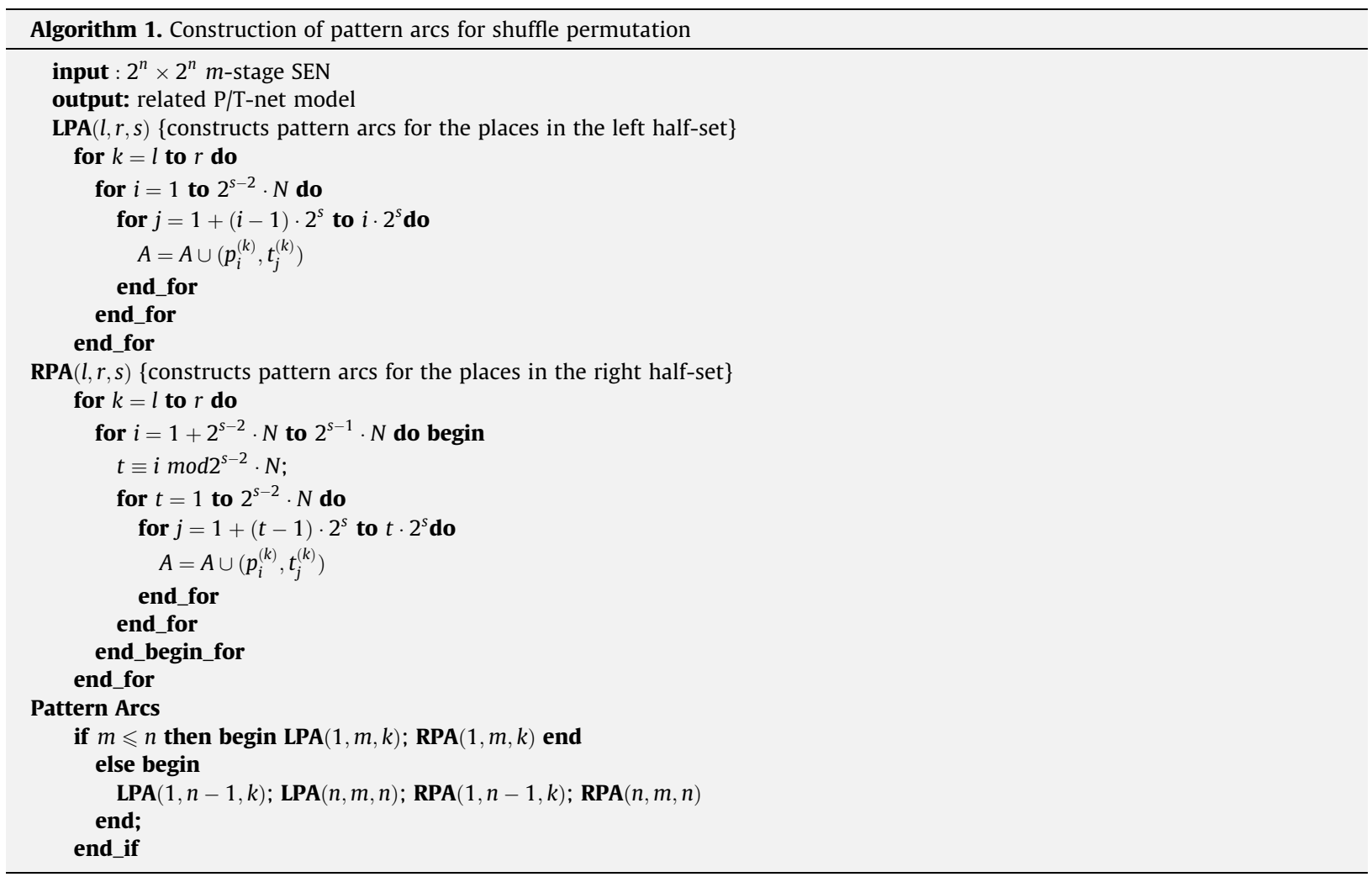


As an example let us consider Algorithm 1 that constructs pattern arcs for OMINs with shuffle permutation between neighboring stages. The construction of pattern arcs progresses in a breadth-first fashion. The algorithm pursues the module LPA in creating the pattern arcs that are incident from places in the leftmost half-set. These arcs are inheritably similar to the shuffle permutation pattern (see Fig. 4). Likewise, the algorithm uses module RPA to create the pattern arcs that are incident from the rightmost half-set places. We obtain all pattern arcs by reusing the modules LPA and RPA in the body of Algorithm 1.

Our P/T-nets are typically represented by sparse block diagonal incidence matrices. The numbers of zero and non-zero elements in these matrices depend on the OMIN size but not OMIN topology. The peculiarities of interstage communication patterns, however, affect the distribution of non-zero elements in the incidence matrices. We derived the distribution of non-zero elements in the incidence matrices corresponding to the P/T-nets of $4 \times 41$ - and 2-stage (Fig. 5), $8 \times 81$-, 2and 3-stage (Fig. 6), and $16 \times 161$-, 2- and 3-stage (Fig. 7) shuffle-exchange OMINs. An interesting observation is that large incidence matrices fully cover smaller ones. In these figures we illustrate all matrices in the range in overlapped fashion starting from the top left corner. For example, Fig. 5 comprises $4 \times 12$ and $20 \times 28$ matrices. In Figs. 5-7, for clarity 1 s and $-1 \mathrm{~s}$ appear with cyan and magenta highlights, respectively.

\section{Complexity and acyclicity}

Given PTNOMIN ${ }_{2^{n}, m}$, we denote the number of transitions and places by $|T|$ and $|P|$, respectively. The following proposition measures the size of $\operatorname{PTNOMIN}_{2^{n}, m}$.

Proposition 1. The following holds:

$$
|\mathscr{P}|= \begin{cases}\left.\left(2^{m+1}-1\right) N\right), & \text { if } 1 \leqslant m<n \\ \frac{1}{2}(m-n+4) N^{2}-N, & \text { if } n \leqslant m<2 n-1,\end{cases}
$$

and

$$
|\mathscr{T}|= \begin{cases}\frac{1}{3}\left(4^{m}-1\right) N, & \text { if } 1 \leqslant m<n \\ \frac{1}{4}\left(m-n+\frac{4}{3}\right) N^{3}-\frac{1}{3} N, & \text { if } n \leqslant m<2 n-1 .\end{cases}
$$

Proof. Due to Definition 2 calculation of $|\mathscr{P}|$ dissipates into ranges $1 \leqslant m<n$ and $n \leqslant m<2 n-1$. When $1 \leqslant m<n$, the number of places in related P/T-net is represented as the sum of the elements of geometric series:

$$
\sum_{k=1}^{m+1}\left|\mathscr{P}_{k}\right|=\sum_{k=1}^{m+1} 2^{k-1} N=\left(2^{0}+2^{1}+\cdots+2^{m}\right) N=\frac{1-2^{m+1}}{1-2} N=\left(2^{m+1}-1\right) N
$$

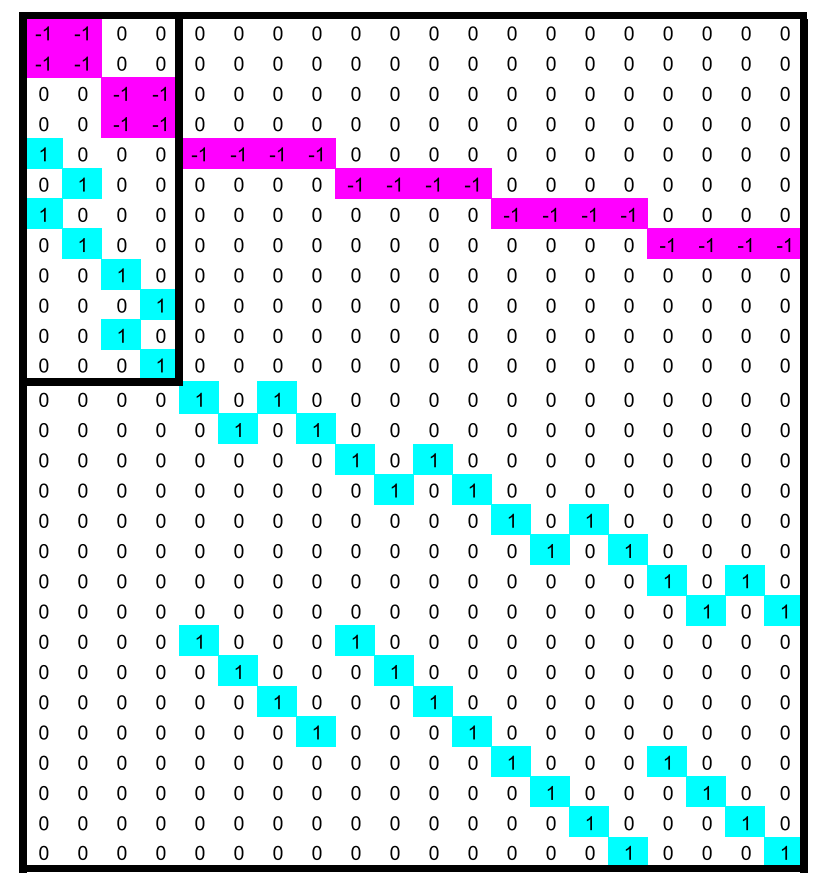

Fig. 5. Incidence matrices for $4 \times 41$ and 2-stage OMINs. 


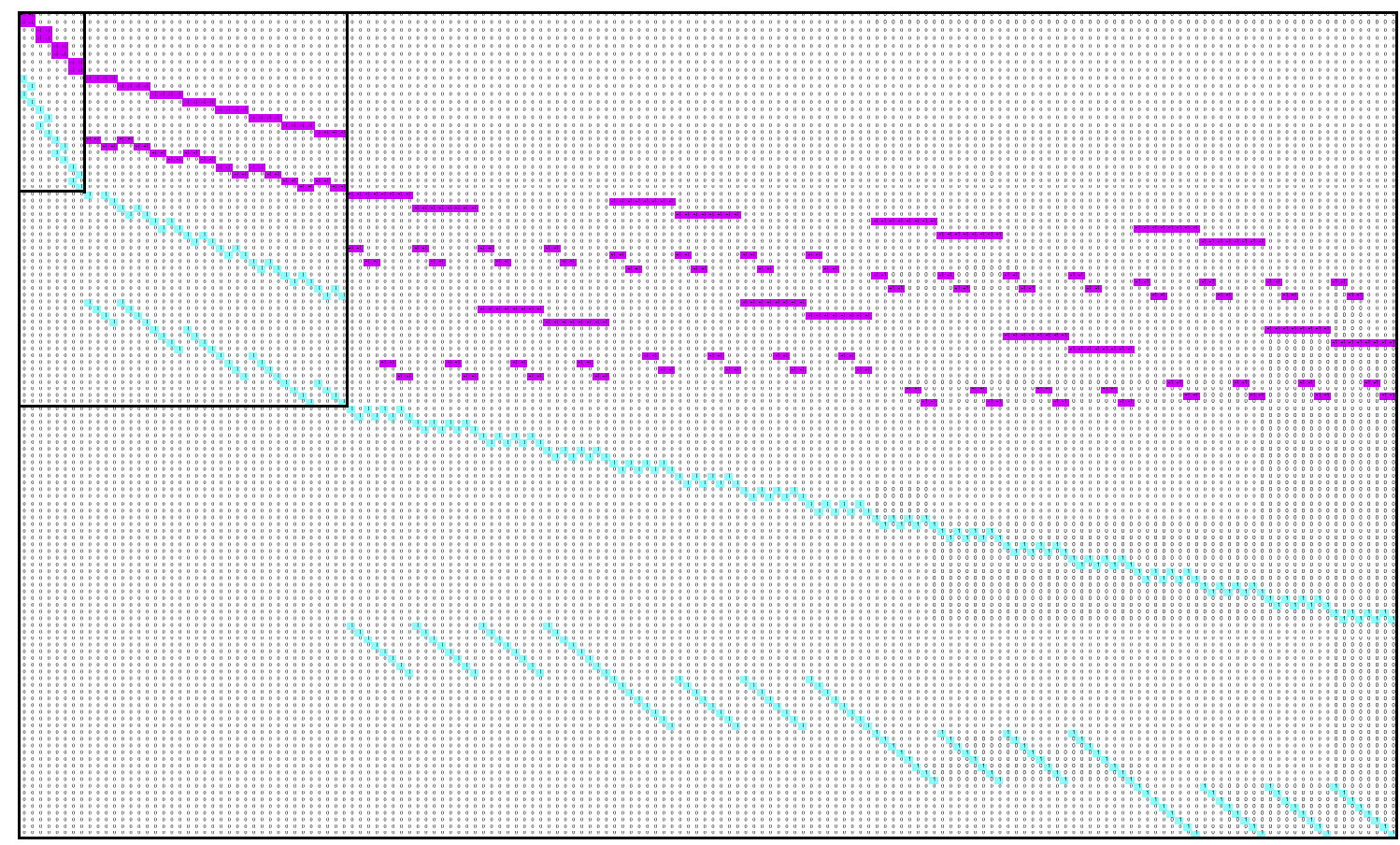

Fig. 6. Incidence matrices for $8 \times 81$ through 3 -stage OMINs.



Fig. 7. Incidence matrices for 1 through 3 -stage $16 \times 16$ OMINs. 
For $n \leqslant m<2 n-1$ the number of places in P/T-net is estimated as follows:

$$
\sum_{k=1}^{m+1}\left|\mathscr{P}_{k}\right|=\sum_{k=1}^{n}\left|\mathscr{P}_{k}\right|+\sum_{k=n+1}^{m+1}\left|\mathscr{P}_{k}\right|=\left(N^{2}-N\right)+\sum_{k=n}^{m+1} 2^{n-1} N=\left(N^{2}-N\right)+\frac{1}{2}(m-n+2) N^{2}=\frac{1}{2}(m-n+4) N^{2}-N .
$$

Combining (3) and (4) we obtain the claim regarding $|\mathscr{P}|$. (6):

The calculation of $|\mathscr{T}|$, that is also carried out separately for $1 \leqslant m<n$ and $n \leqslant m<2 n-1$, is given by formulae (5) and

$$
\begin{aligned}
\sum_{k=1}^{m}\left|\mathscr{T}_{k}\right|= & \sum_{k=1}^{m} 4^{k-1} N=\left(4^{0}+4^{1}+\cdots+4^{m-1}\right) N=\frac{1-4^{m}}{1-4} N=\frac{1}{3}\left(4^{m}-1\right) N . \\
\sum_{k=1}^{m}\left|\mathscr{T}_{k}\right|= & \sum_{k=1}^{n-1}\left|\mathscr{T}_{k}\right|+\sum_{k=n}^{m}\left|\mathscr{T}_{k}\right|=\left(\frac{1}{12} N^{3}-\frac{1}{3} N\right)+\sum_{k=n}^{m} 4^{n-1} N=\left(\frac{1}{12} N^{3}-\frac{1}{3} N\right) \\
& +\frac{1}{4}(m-n+1) N^{3}=\frac{1}{4}\left(m-n+\frac{4}{3}\right) N^{3}-\frac{1}{3} N .
\end{aligned}
$$

Combining (5) and (6), we obtain the exact order for $|\mathscr{T}|$, which agrees with the claim.

The size of PTNOMIN $2^{n}, m$ is directly proportional to the memory capacity that is used to store it. It is quite often the case that a P/T-net model which is exponential in the size of the original problem is built even for a modest-size problem [9]. Proposition 1 establishes the exact orders for $|\mathscr{P}|$ and $|\mathscr{T}|$, which are in polynomial dependence on the problem size $N$. Polynomial behavior of PTNOMIN $2_{2^{n}, k}$ allows us to avoid memory overflow and supports the fact that memory capabilities and the performance of modern computers are indeed sufficient to run our task.

In [1] we created a CP-net model of OMIN and converted resulting CP-nets into complete unfoldings. The size of complete unfoldings are represented by polynomial functions $\left|\mathscr{P}_{U n f}\right|=\frac{(m+2) N^{2}}{2}+\frac{m N}{2}$ and $\left|\mathscr{T}_{U n f}\right|=\frac{m N^{3}}{2}$.

The following proposition assesses the relative merits of the aforementioned complete unfoldings and equivalent $\mathrm{P} / \mathrm{T}$ nets regarding their complexity characteristics.

Proposition 2. The following is valid:

$$
|\mathscr{P}|<\left|\mathscr{P}_{\text {Unf }}\right| \text { and }|\mathscr{T}|<\left|\mathscr{T}_{\text {Unf }}\right| \text {. }
$$

Proof. We prove the claim through assessing the ratios $\frac{|\mathscr{P}|}{\left.\right|^{\mathscr{P}_{\text {Unf }} \mid} \mid}$ and $\frac{|\mathscr{T}|}{\left|\mathscr{F}_{\text {Unf }}\right|^{*}}$.

For $1 \leqslant m<n$ we have

$$
\frac{|\mathscr{P}|}{\left|\mathscr{P}_{\text {Unf }}\right|}=\frac{2\left(2^{m+1}-1\right) N}{(m+2) N^{2}+m N}=\frac{2\left(2^{m+1}-1\right)}{(m+2) N+m} \leqslant \frac{2 N-2}{(m+2) N+m}<1
$$

and

$$
\frac{|\mathscr{T}|}{\left|\mathscr{T}_{\text {Unf }}\right|}=\frac{2\left(4^{m}-1\right) N}{3 m N^{3}}<\frac{2 N^{3}-2 N}{3 m N^{3}}=\frac{2-2 / N^{2}}{3 m}<1
$$

When $n \leqslant m<2 n-1$ we obtain

$$
\frac{|\mathscr{P}|}{\left|\mathscr{P}_{U n f}\right|}=\frac{(m-n+4) N^{2}-2 N}{(m+2) N^{2}+k N}=\frac{m-n+4-2 / N}{m+2+k / N}<1
$$

and

$$
\frac{|\mathscr{T}|}{\left|\mathscr{T}_{\text {Unf }}\right|}=\frac{(3 m-3 n+4) N^{3}-4 N}{6 m N^{3}}=\frac{3 m-3 n+4-4 / N^{2}}{6 m}<1
$$

Thus, $|\mathscr{P}|<\left|\mathscr{P}_{\text {Unf }}\right|$ and $|\mathscr{T}|<\left|\mathscr{T}_{\text {Unf }}\right|$, as claimed.

This result is as expected, as each $\mathrm{P} / \mathrm{T}$-net created in the present work is, in fact, an optimized complete unfolding which is drawn from scratch based on OMIN specifications rather than obtained through optimizing related complete unfolding. Each optimized complete unfolding, however, is inevitably more compact since it is obtained from an original complete unfolding by removing unnecessary components such as 0-bounded places, false-guarded transitions, etc.

Proposition 3. PTNOMIN $2^{n}, m$ is an acyclic net.

Proof. The proof is straightforward from Definition 2, and the construction of the complete occurrence sequence (2). 
Table 1

$4 \times 4$ OMIN: results of computer experiments.

\begin{tabular}{lll}
\hline Permutations & $m_{\min }$ & Time/in second \\
\hline$\pi_{1}=\left(\begin{array}{llll}0 & 1 & 2 & 3 \\
0 & 1 & 3 & 2\end{array}\right)$ & 1 \\
$\pi_{2}=\left(\begin{array}{llll}0 & 1 & 2 & 3 \\
1 & 3 & 2 & 0\end{array}\right)$ & 2 \\
\hline
\end{tabular}

Table 2

$8 \times 8$ OMIN: results of computer experiments.

\begin{tabular}{|c|c|c|c|c|c|c|c|c|c|}
\hline \multicolumn{8}{|c|}{ Permutations } & \multirow{2}{*}{$\frac{m_{\min }}{4}$} & \multirow{2}{*}{$\begin{array}{l}\text { Time/in second } \\
0.06\end{array}$} \\
\hline$\pi_{3}=($ & $\begin{array}{l}1 \\
6\end{array}$ & $\begin{array}{l}2 \\
2\end{array}$ & $\begin{array}{l}3 \\
3\end{array}$ & $\begin{array}{l}4 \\
0\end{array}$ & $\begin{array}{l}5 \\
1\end{array}$ & $\begin{array}{l}6 \\
4\end{array}$ & $\left.\begin{array}{l}7 \\
5\end{array}\right)$ & & \\
\hline$\pi_{4}=($ & $\begin{array}{l}1 \\
0\end{array}$ & $\begin{array}{l}2 \\
2\end{array}$ & $\begin{array}{l}3 \\
3\end{array}$ & $\begin{array}{l}4 \\
5\end{array}$ & $\begin{array}{l}5 \\
4\end{array}$ & $\begin{array}{l}6 \\
7\end{array}$ & $\left.\begin{array}{l}7 \\
6\end{array}\right)$ & 1 & 0.01 \\
\hline$\pi_{5}=$ & $\begin{array}{l}1 \\
3\end{array}$ & $\begin{array}{l}2 \\
4\end{array}$ & $\begin{array}{l}3 \\
6\end{array}$ & $\begin{array}{l}4 \\
1\end{array}$ & $\begin{array}{l}5 \\
2\end{array}$ & $\begin{array}{l}6 \\
5\end{array}$ & $\left.\begin{array}{l}7 \\
7\end{array}\right)$ & 2 & 0.02 \\
\hline$\pi_{6}=$ & $\begin{array}{l}1 \\
0\end{array}$ & $\begin{array}{l}2 \\
1\end{array}$ & $\begin{array}{l}3 \\
5\end{array}$ & $\begin{array}{l}4 \\
2\end{array}$ & $\begin{array}{l}5 \\
4\end{array}$ & $\begin{array}{l}6 \\
3\end{array}$ & $\left.\begin{array}{l}7 \\
6\end{array}\right)$ & 3 & 0.03 \\
\hline$\pi_{7}=$ & $\begin{array}{l}1 \\
2\end{array}$ & $\begin{array}{l}2 \\
4\end{array}$ & $\begin{array}{l}3 \\
6\end{array}$ & $\begin{array}{l}4 \\
0\end{array}$ & $\begin{array}{l}5 \\
2\end{array}$ & $\begin{array}{l}6 \\
5\end{array}$ & $\left.\begin{array}{l}7 \\
7\end{array}\right)$ & 2 & 0.03 \\
\hline
\end{tabular}

Table 3

$16 \times 16$ OMIN: results of computer experiments.

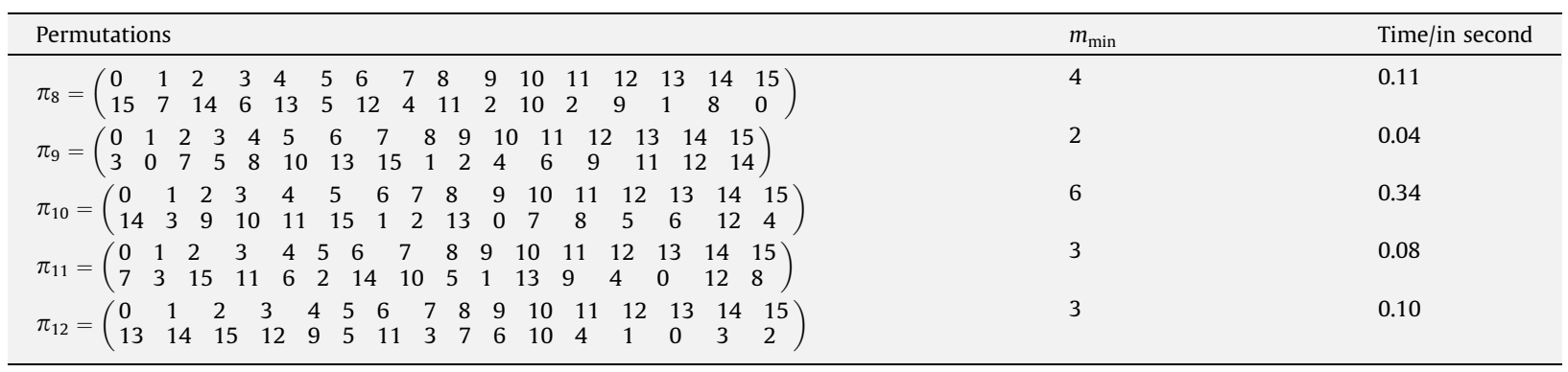

\section{Experimental results}

We have tested the effectiveness of our approach through a series of computer experiments. We have determined $m_{\min }$ for $4 \times 4,8 \times 8$, and $16 \times 16$ shuffle-exchange OMINs in the realization of randomly chosen permutations. Computer experiments were performed in a high-level interactive environment Matlab on a Windows XP platform with $3 \mathrm{GHz}$ of CPU frequency and 4 GB RAM. The results of computer experiments are illustrated in Tables 1-3.

We are aware of the fact that the incidence matrices (see Figs. 5-7), as well as marking vectors obtained in present research, are sparse in nature, and that there exist dozens of effective sparse matrix methods that can be used to solve linear algebraic equations. We did not attempt any of those methods, however, since the most time consuming case of $\pi_{10}$ (see Table 3 ) took only $0.34 \mathrm{~s}$ to complete the task with the Matlab standard library function.

We wrote an interface program in C++ to convert input data into a format suitable for Matlab, viz. a rectangular table of numbers. It should be stressed that a result of "no solution" was obtained almost at no time. So, any time value indicated in Tables $1-3$ is indeed the run time of the task for $m_{\min }$ rather than the cumulative run time for all cases 1 through $m_{\min }$. Considering again the example of $\pi_{10}, 0.34 \mathrm{~s}$ is mainly the time spent to check admissibility of $\pi_{10}$ to 6 -stage OMIN.

The comparison of run times obtained in the present research and those obtained in [1] supports the time-effectiveness of this work. For all instances the present approach takes much less time.

\section{Conclusions}

This paper explores interaction between Petri nets and optical communication, to the benefit of both fields. On the one hand we adopt Petri nets for problem solving in optical communication, and consequently widen the spectrum of their applications; on the other hand, we deal with two critical problems arising in optical communication, viz. path dependent loss and switch crosstalks. 
The main results obtained are summarized as follows: (1) a Petri nets based approach is proposed to reduce path dependent loss and the number of switch crosstalks in OMINs; (2) it is easy to create a Petri net model; (3) Petri nets, that are created in the present research, are in polynomial dependence on the OMIN size which minimizes the memory consumption; (4) these Petri nets are acyclic which allows us to use P-invariants for reachability analysis; (5) complexity results obtained in [1] have improved; and (6) results of computer experiments demonstrate the time-effectiveness of the proposed approach.

\section{References}

[1] R. Bashirov, F. Kordon, H. Lort, Exploiting colored Petri nets to decide on permutation admissibility, Acta Informatica 46 (2009) $43-55$.

[2] R. Bashirov, V. Crespi, Analyzing the permutation capability of multistage interconnection networks with colored Petri nets, Information Sciences 176 (2006) 3143-3165.

[3] D. Benhaddou, G. Chaudhry, Photonic switching techniques and architecture for next generation optical networks, Cluster Computing 7 (2004) 281291.

[4] N. Das, B.B. Bhattacharya, S. Bezrukov, R. Menon, A. Sarkar, Permutation routing in optical MINs with minimum number of stages, Journal of Systems Architecture 48 (2003) 311-323.

[5] Y.Y. Du, C.J. Jiang, M.C . Zhou, Y. Fu, Modelling and monitoring of E-commerce workflows, Information Sciences 179 (2009) $995-1006$.

[6] L. Guo, X. Wang, Q. Song, X. Wei, W. Hou, T. Yang, F. Yang, New insights on survivability in multidomain optical networks, Information Sciences 178 (2008) 3635-3644.

[7] X. Jiang, H. Sheng, M.R. Khandker, S. Horiguchi, Blocking behaviors of crosstalk-free optical banyan networks on vertical stacking, IEEE/ACM Transactions on Networking 11 (2003) 982-993.

[8] A.K. Katangur, S. Akkaladevi, Y. Pan, Analyzing the performance of optical multistage interconnection networks with limited crosstalk, Cluster Computing 10 (2007) 241-250.

[9] V. Khomenko, A. Kondratyev, A. Koutny, W. Vogler, Merged processes: a new condenced representation of Petri net behaviour, Acta Informatica 43 (2006) 307-330.

[10] C. Lea, Crossover minimization in directional-coupler-based photonic switching systems, IEEE Transactions on Communications 36 (1988) 355-363.

[11] D.C. McCarthy, Photonic Switches: Fast, But Functional, Photonics Spectra (2001) 141-150. March.

[12] T. Murata, Petri nets: properties, analysis and applications, Proceedings of IEEE 77 (1989) 541-580.

[13] I. O'Connor, Optical solutions for system level interconnect, in: Proceedings of the SLIP'2004, Paris, 14-15 February, 2004, pp. 79-88.

[14] R.G. Özdemir, G. Gençyılmaz, T. Aktin, The modified fuzzy art and a two-stage clustering approach to cell design, Information Sciences 177 (2007) 5219-5236.

[15] K. Padmanabhan, A.N. Netravali, Dilated networks for switching, IEEE Transactions on Communications 35 (1987) $1357-1365$.

[16] Yi Pan, Optical multistage interconnection networks: new challenges and approaches, IEEE Communications Magazine 37 (1999) 50-56.

[17] S. Sahni, Matrix multiplication and data routing using a partitioned optical passive stars networks, IEEE Transactions on Parallel and Distributed Computing 11 (2000) 720-728.

[18] X. Shen, An optimal $O(N \lg N)$ algorithm for permutation admissibility to extra-stage cube-type networks, IEEE Transactions on Computers 44 (1995) 1144-1149.

[19] X. Shen, F. Yang, F. Pan, Equivalent permutation capabilities between time-division optical omega networks and non-optical extra-stage omega networks, IEEE Transactions on Networking 9 (2001) 518-524.

[20] X. Shen, M. Xu, X. Wang, An optimal algorithm for permutation admissibility to multistage interconnection networks, IEEE Transactions on Computers 44 (1995) 604-608

[21] X.F. Shen, Y. Pan, Equivalent permutation capabilities between time-division optical omega networks and non-optical extra-stage omega networks, IEEE Transactions on Networking 9 (2001) 518-524.

[22] H. Tian, A.K. Katangur, J. Zhong, Y. Pan, A novel multistage architecture with multicast and broadcast capability, The Journal of Supercomputing 35 (2006) 277-300.

[23] M.M. Vaez, C.T. Lea, Strictly nonblocking directional-coupler based switching networks under crosstalk constraint, IEEE Transactions on Communication 48 (2000) 316-323.

[24] M.T. Wynn, H.M.W. Verbeek, W.M.P. van der Aalst, A.H.M. ter Hofstede, D. Edmond, Soundness-preserving reduction rules for reset workflow nets, Information Sciences 179 (2009) 769-790.

[25] Y. Yang, J. Wang, Y. Pan, Permutation capability of optical interconnection networks, Journal of Parallel and Distributed Computing 60 (2000) $72-91$. 